\title{
IMPROVMENT OF GROWTH AND QUALITY AND REGULATION OF THE ANTIOXIDANT SYSTEM AND LIPID PEROXIDATION IN CHINESE CABBAGE (Brassica pekinensis (Lour.) Rupr.) BY EXOGENOUS SODIUM SELENITE
}

\author{
LIU, L. - WANG, L. X. ${ }^{*}$ - LV, L. H. - WeI, J. P. - LIU, Q. L. - CHENG, L. - QU, J. \\ Heze Academy of Agricultural Sciences, Shandong Province, Heze 274000, PR China \\ *Corresponding author \\ sddt1401@126.com
}

(Received 15 $5^{\text {th }}$ Dec 2019; accepted 26 $6^{\text {th }}$ Mar 2020)

\begin{abstract}
Selenium (Se) is a necessary mineral nutrient for humans. In order to study the effects of exogenous sodium selenite on Chinese cabbage, an important food in people's daily life, the present study was conducted with four applications with different concentrations of sodium selenite, 0 (CK), 50 (Se1), 150 (Se2), 250 (Se3) $\mathrm{mg} \mathrm{L}^{-1}$. The results showed that the concentrations of Se, Fe and $\mathrm{Zn}$ in Chinese cabbage increased due to the Se application while the fresh weight and dry weight were affected beneficially by the treatment of Se just like chlorophyll contents. Foliar application of sodium selenite also remarkably increased the $\mathrm{Vc}$, proline, protein and sugar contents. Furthermore, the foliar application of Se remarkably enhanced antioxidant enzyme activities of POD, SOD, CAT, APX and GR. In conclusion, exogenous sodium selenite would improve the growth and quality and enhance antioxidant resistance of Chinese cabbage.
\end{abstract}

Keywords: chlorophyll, vegetable, selenium, crop quality, antioxidant enzymes

\section{Introduction}

Selenium (Se) is one of the essential trace elements for human and animal growth. $72 \%$ of the Chinese counties (cities) are selenium deficient or low selenium areas, and two-thirds of the population suffer from selenium malnutrition or selenium deficiency (Tan et al., 2002; Xia et al., 2005). Previous research studies have proved that proper selenium supplementation is an effective measure to enhance human health, prevent diseases and prolong life (Rayman, 2000; Yang et al., 2017). Plants are the main source of selenium for humans and animals, thus the production of selenium - rich plants is an effective way to supplement human selenium.

As an important vegetable for the life of Chinese, Chinese cabbage (Brassica pekinensis (Lour.) Rupr.) is an indispensable delicious vegetable on the dining table of urban and rural residents in autumn and winter. Chinese cabbage not only possesses high contents of dry substances, vitamin $\mathrm{C}(\mathrm{Vc})$, protein and soluble sugar, but also has low acidity and crude fiber (Zhang et al., 2002; Su et al., 2018). Chinese cabbage also had a long history of cultivation and a large cultivated area in China (De Medici et al., 2019; Mi et al., 2019). Some previous studies have shown the beneficial effect of exogenous Se on Chinese cabbage performances. For example, the study of $\mathrm{Wu}$ et al. (2018) revealed that foliar application of Se selectively induced glutathione reductase (GR) and dehydroascorbic acid reductase (DHAR) in Chinese cabbage tissues and mitigated the cadmium toxicity. Dai et al. (2019) also used a year field experiment to find an interaction effect between soil application of Se and Zinc ( $\mathrm{Zn})$ on Chinese cabbage quality and antioxidant system through a pot experiment. However, the foliar field application of $\mathrm{Se}$ on Chinese cabbage was rarely reported the utilization rate of 
microelements would be much higher in foliar application than in soil application (Farooq et al., 2018).

Therefore, in order to improve the scientific database on factors affecting the quality and yield of Chinese cabbage and enhance the application of Se in Chinese cabbage production, present field experiment was conducted in Shandong province, China, in 2019 with two hypotheses, that are (1) foliar application of selenite would improve the growth and development of Chinese cabbage and (2) foliar application of selenite would promote the quality of Chinese cabbage.

\section{Materials and Methods}

\section{Experimental details}

The field experiment was conducted in Heze city of Shandong province, China. The experimental soil is sandy loam soil and the $\mathrm{pH}$ value was 6.7 , and with $25.99 \mathrm{~g} \mathrm{~kg}^{-1}$ total nitrogen, $2.44 \mathrm{~g} \mathrm{~kg}^{-1}$ available phosphorus and $16.90 \mathrm{mg} \mathrm{kg}^{-1}$ available potassium. Four concentrations of sodium selenite were applied as follows: overhead sprinkling with 0 , 50, 150, and $250 \mathrm{mg} \cdot \mathrm{L}^{-1}$ and these treatments were known as CK, Se1, Se2, Se3, respectively. The treatments were arranged in a randomized complete block design (RCBD) in triplicate. One day before harvest, three representative plants of each treatment were collected for the bio-chemical indexes including nutritional components, antioxidative enzymes, chlorophyll, malondialdehyde, Se, Fe and $\mathrm{Zn}$. At harvest, ten random Chinese cabbage plants were collected from each treatment to measure the fresh weight and dry weight.

\section{Estimation of antioxidative enzymes, malondialdehyde and chlorophyll}

The activities of catalase (CAT), superoxide dismutase (SOD), peroxidase (POD) and the malondialdehyde (MDA) were measured according to the methods described by Luo et al. (2018). The chlorophyll $\mathrm{a}$, chlorophyll $\mathrm{b}$ and carotenoid contents were estimated by the methods of $\mathrm{He}$ et al. (2019). The activities of ascorbate peroxidase (APX) and glutathione reductase (GR) were determinate according to Dai et al. (2015).

\section{Estimation of nutritional components of Chinese Cabbage}

The protein and proline contents were determinate by the methods of $\mathrm{Li}$ et al. (2016) using Coomassie brilliant blue G-250 and ninhydrin colorimetry, respectively. The content of vitamin $\mathrm{C} \quad(\mathrm{Vc})$ was estimated through titration with 2,6-dichlorophenolindophenol and the sugar content was measured by anthrone method according to Ma et al. (2014).

\section{Determination of Se, Fe, Zn contents}

The plant samples were ground into powder form and digested $(0.1 \mathrm{~g})$ with $1.5 \mathrm{ml}$ of concentrated $\mathrm{HNO}_{3}$ and $0.5 \mathrm{ml} 30 \% \mathrm{H}_{2} \mathrm{O}_{2}$ in a digestive stove at $180^{\circ} \mathrm{C}$ for $1.5 \mathrm{~h}$. Total $\mathrm{Se}, \mathrm{Fe}$ and $\mathrm{Zn}$ content in samples were determined by using atomic absorption spectrometer (Z2300, HITACHI, Japan)-HFS (HFS-3, HITACHI, Japan). 


\section{Statistical analysis}

Data were analyzed on Statistix 8.1 (Analytical Software, Tallahassee, FL, USA) while differences among means were separated by using least significant difference (LSD) test at 5\% probability level. Graphical representation was conducted via Sigma Plot 14.0 (Systat Software Inc., California, USA).

\section{Results}

\section{Biomass}

Foliar application of sodium selenite had impacts on fresh weight and dry weight of Chinese cabbage (Figure 1). Compared with CK, Se1 treatment significantly increased the fresh weight and dry weight by 36.26 and $47.70 \%$, respectively. However, there was no remarkable difference observed among CK, Se 2 and Se3 in both fresh weight and dry weight of Chinese cabbage.
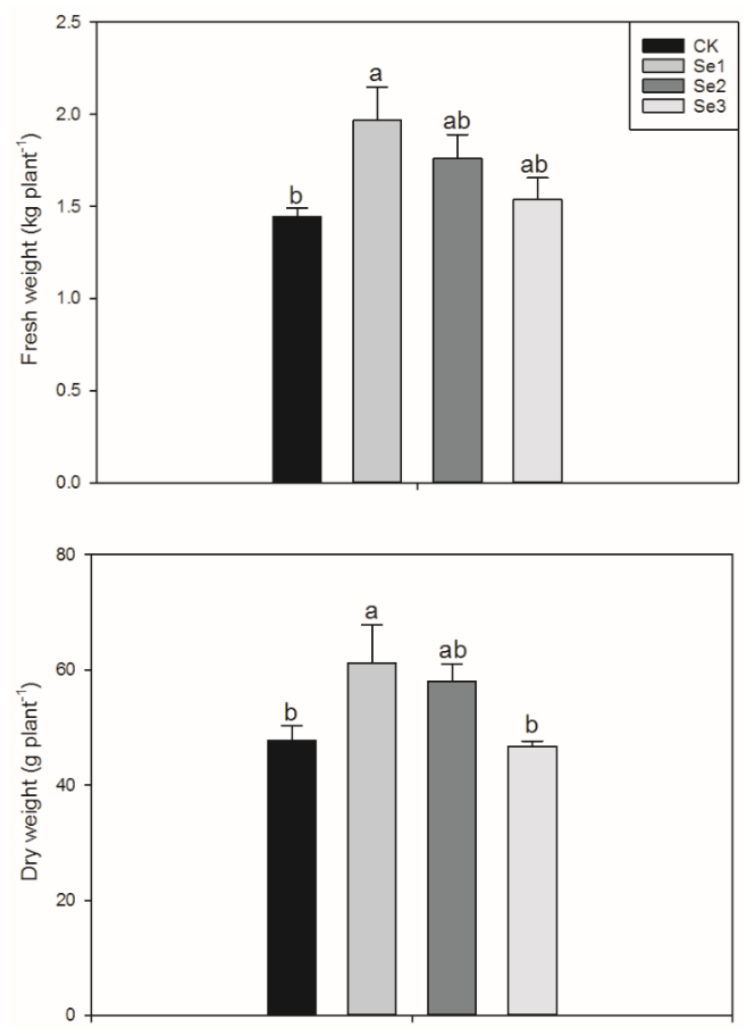

Figure 1. Effects of exogenous sodium selenite on fresh weight and dry weight in Chinese cabbage. Capped bars represent S.E. of three replicates. Means sharing a common letter do not differ significantly at $(P \leq 0.05)$ according to least significant difference (LSD) test

\section{Antioxidative enzymes and lipid peroxidation}

Foliar application of sodium selenite regulated the activities of POD, SOD, CAT, GR, APX and contents of MDA in Chinese cabbage (Figure 2). Compared to CK, Se1, Se2 and Se3 treatments significantly increased POD activities by $103.36 \%, 56.83 \%$ and $16.63 \%$, respectively. For SOD activity, $31.86 \%$ and $12.67 \%$ higher activities were 
recorded in $\mathrm{Se} 1$ and $\mathrm{Se} 2$ treatments than in $\mathrm{CK}$ whiles there was no remarkable difference between $\mathrm{CK}$ and $\mathrm{Se} 3$. Compared to $\mathrm{CK}, \mathrm{Se} 1, \mathrm{Se} 2$ and $\mathrm{Se}$ treatments significantly increased CAT activities by $104.32 \%, 51.57 \%$ and $13.10 \%$, respectively. For APX activities, $190.78 \%, 101.59 \%$ and $39.15 \%$ higher activities were recorded in Se1, Se2 and $\mathrm{Se} 3$ treatments compared with CK, respectively. Compared to CK, Se1, Se2 and $\mathrm{Se} 3$ treatments significantly increased GR activities by $120.71 \%, 63.71 \%$ and $20.95 \%$, respectively. For the contents of MDA, Se1, Se2 and $\mathrm{Se} 3$ treatments significantly increased MDA contents compared with $\mathrm{CK}$ whilst there was no remarkable difference among Se1, Se 2 and $\mathrm{Se} 3$ in MDA contents.
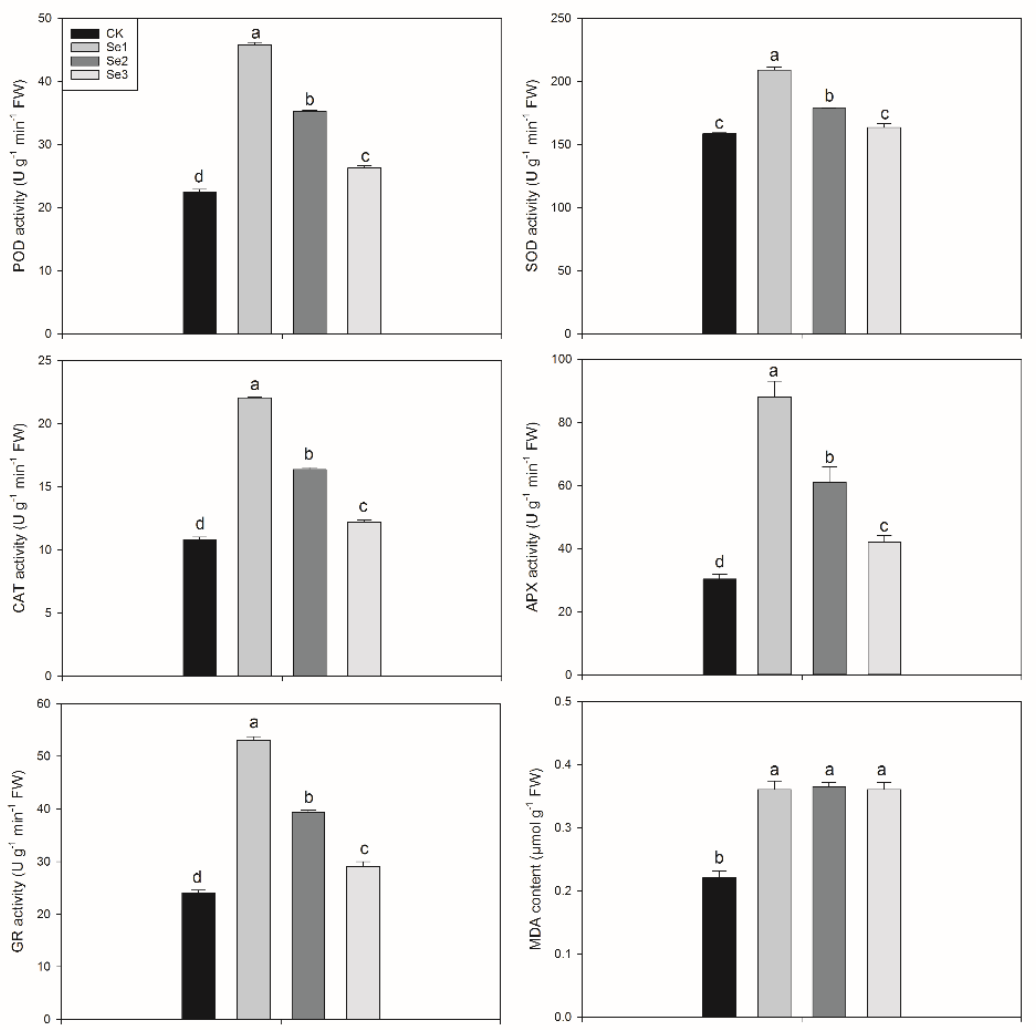

Figure 2. Effects of exogenous sodium selenite on activities of POD, SOD, CAT, GR, APX and contents of MDA in Chinese cabbage. Capped bars represent S.E. of three replicates. Means sharing a common letter do not differ significantly at $(P \leq 0.05)$ according to least significant difference (LSD) test

\section{Chlorophyll content}

As shown in Figure 3, foliar application of sodium selenite significantly affected the contents of chlorophyll a, chlorophyll $b$ and carotenoids in Chinese cabbage. Compared with $\mathrm{CK}, \mathrm{Se} 1, \mathrm{Se} 2$ and $\mathrm{Se} 3$ treatments all significantly increased the chlorophyll a content while the highest content was recorded in Se1 treatment. Similar trends were also found in both chlorophyll $\mathrm{b}$ and carotenoids contents.

\section{Nutritional components}

Foliar application of sodium selenite regulated the contents of proline, protein, Vc and sugar in Chinese cabbage (Figure 4). Compared to $\mathrm{CK}$, Se1 and Se2 significantly 
increased proline content by $40.16 \%$ and $26.18 \%$, respectively. For Vc content, $27.07 \%$, $17.56 \%$ and $10.46 \%$ higher contents were recorded in $\mathrm{Se} 1, \mathrm{Se} 2$ and $\mathrm{Se} 3$ treatments than in $\mathrm{CK}$, respectively. Compared to $\mathrm{CK}, \mathrm{Se} 1, \mathrm{Se} 2$ and $\mathrm{Se}$ treatments significantly increased protein content by $48.43 \%, 31.08 \%$ and $18.12 \%$, respectively. For sugar content, $65.97 \%$, $45.00 \%$ and $25.44 \%$ higher values were recorded in $\mathrm{Se} 1, \mathrm{Se} 2$ and $\mathrm{Se} 3$ treatments compared with CK.
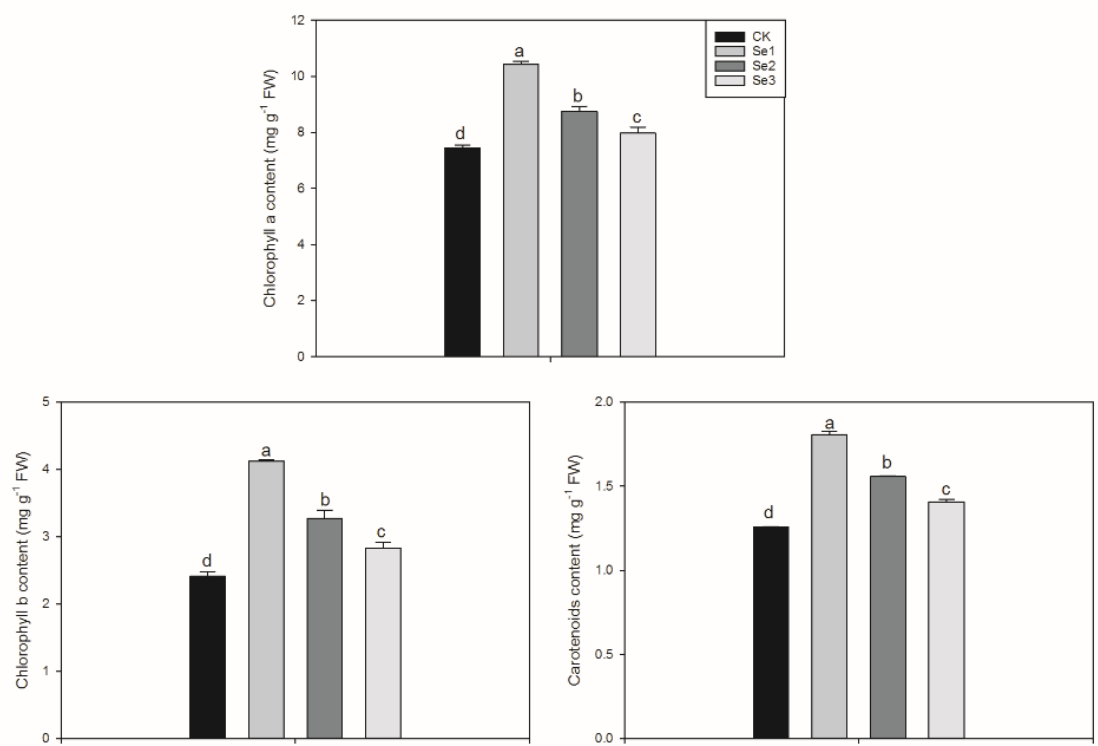

Figure 3. Effects of exogenous sodium selenite on contents of chlorophyll a, chlorophyll b and carotenoids in Chinese cabbage. Capped bars represent S.E. of three replicates. Means sharing a common letter do not differ significantly at $(P \leq 0.05)$ according to least significant difference
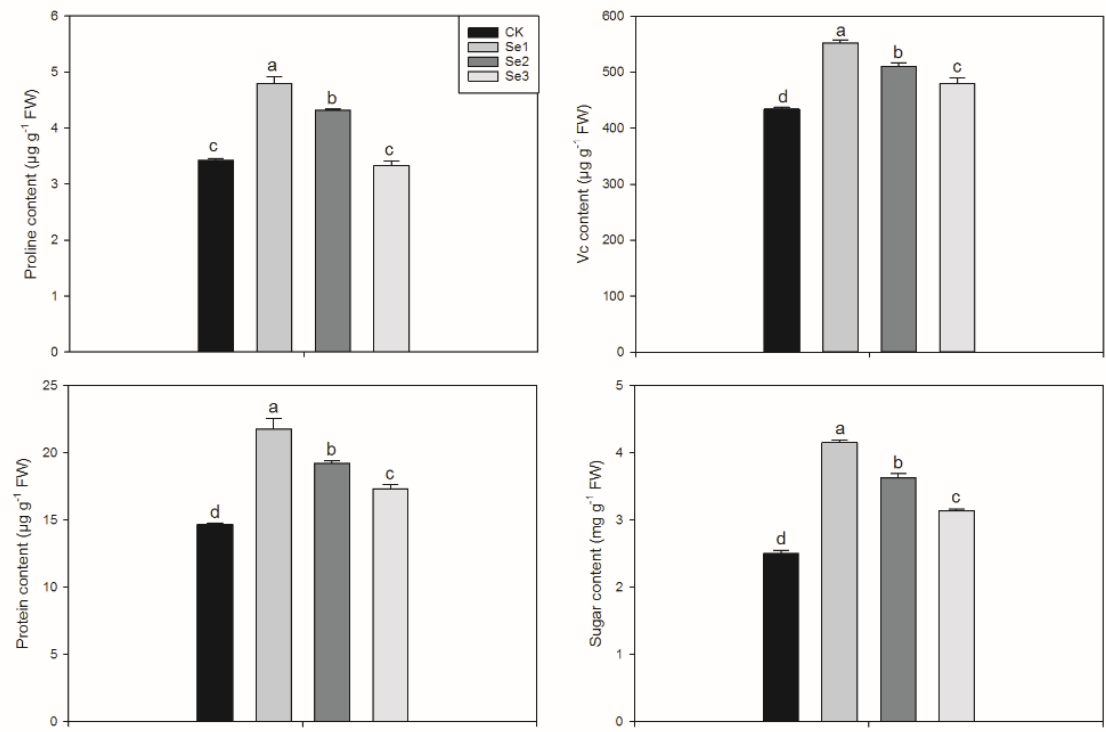

Figure 4. Effects of exogenous sodium selenite on contents of proline, protein, Vc and sugar in Chinese cabbage. Capped bars represent S.E. of three replicates. Means sharing a common letter do not differ significantly at $(P \leq 0.05)$ according to least significant difference (LSD) test 


\section{Se, Fe and Zn content}

As shown in Figure 5, foliar application of sodium selenite has some impacts on Se, $\mathrm{Fe}$ and $\mathrm{Zn}$ concentrations in Chinese cabbage. The Se content increased with the increment of Se applied concentrations and the highest content was recorded in Se3 treatment. However, the Fe content showed a different trend compared to Se. The highest content of Fe was recorded in Se1 treatment while the trend was recorded as: $\mathrm{Se} 1>\mathrm{Se} 2$ $>\mathrm{Se} 3>\mathrm{CK}$. Similar trend was also observed in Zn content.
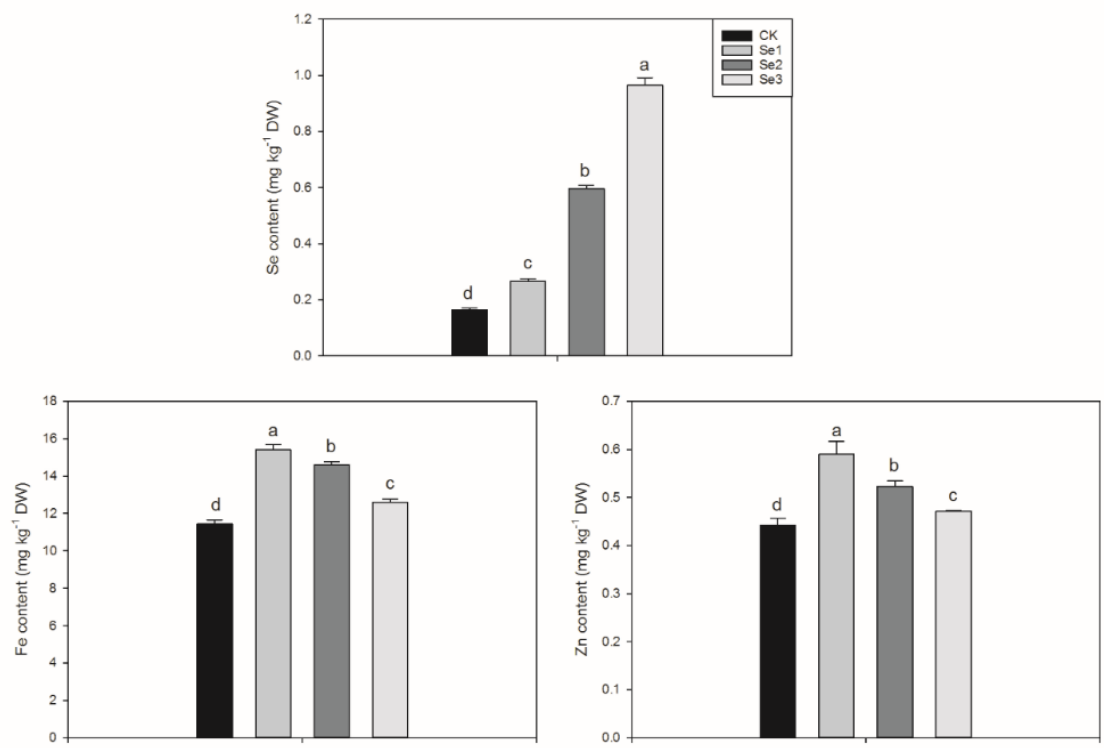

Figure 5. Effects of exogenous sodium selenite on Se, Fe and Zn content in Chinese cabbage. Capped bars represent S.E. of three replicates. Means sharing a common letter do not differ significantly at $(P \leq 0.05)$ according to least significant difference (LSD) test

\section{Analysis of variance}

As shown in Table 1, foliar application of Se had significant effects on activities of POD, SOD, CAT, APX, GR as well as MDA content. The contents of chlorophyll a, chlorophyll $\mathrm{b}$ and carotenoid were significantly affected by Se application. In addition, proline, Vc, protein and sugar contents and contents of Se, Fe and Zn were significantly affected by Se application. However, foliar application of Se had no significant effect on fresh weight and dry weight of Chinese cabbage.

Table 1. Variance analysis about the effects of Se on Chinese cabbage performances

\begin{tabular}{cc|cc}
\hline Parameter & $\boldsymbol{F}$ values & Parameter & $\boldsymbol{F}$ values \\
\hline Fresh weight & $2.61 \mathrm{~ns}$ & Chlorophyll b & $58.45^{* *}$ \\
Dry weight & $3.04 \mathrm{~ns}$ & Carotenoid & $306.39 * *$ \\
POD & $817.81^{* *}$ & Proline & $66.92 * *$ \\
SOD & $144.74 * *$ & Vc & $120.95 * *$ \\
CAT & $802.02^{* *}$ & Protein & $41.59 * *$ \\
APX & $35.53 * *$ & Sugar & $199.71 * *$ \\
GR & $465.57 * *$ & Se content & $455.59 * *$ \\
MDA & $37.32^{* *}$ & Fe content & $76.01 * *$ \\
Chlorophyll a & $78.15^{* *}$ & Zn content & $20.63 * *$ \\
\hline
\end{tabular}

$F$ value and significance level $(* * \mathrm{P}<0.01, * \mathrm{P}<0.05$ and $\mathrm{ns} \mathrm{P} \geq 0.05)$ 


\section{Discussion}

Present study made the first attempt about the foliar application of Se in Chinese cabbage production and revealed the beneficial effects of exogenous Se on biomass, photosynthetic pigments, quality and antioxidant system of Chinese cabbage. The results supported both hypothesizes that foliar application of sodium selenite improves growth and development as well as quality of Chinese cabbage. At the most fundamental level, foliar application of sodium selenite increased the Se content in Chinese cabbage and the Se content increased with the increment in the applied concentrations. On the other hand, the foliar application of Se significantly increased both $\mathrm{Zn}$ and Fe contents and the highest $\mathrm{Zn}$ content and Fe content were both recorded in Se1 treatment. This result indicated that exogenous Se would enhance the absorption of Chinese cabbage to $\mathrm{Zn}$ and Fe which was consistent with the studies of Dai et al. (2019) and Fang et al. (2008).

As expected, foliar application of sodium selenite ( $\mathrm{Se} 1$ treatment) produced the Chinese cabbage with higher fresh weight and dry weight. The biomass was enhanced probably due to the increments in photosynthetic pigment contents including chlorophyll a, chlorophyll b and carotenoids. Our results agreed with the study of Lai et al. (2019) who demonstrated that foliar application of Se promoted the yield of fragrant rice by increasing the chlorophyll content and enhancing photosynthesis. The investigation of Wu et al. (2000) also showed that exogenous Se applied with low concentration would promote the electron transfer rate of chloroplast in paddy rice.

Besides yield, the quality of Chinese cabbage is a determinant factor in economic returns for farmers. The quality of Chinese cabbage is influenced by parameters including protein, sugar, Vc and amino acid such as proline. Present study revealed that foliar application of sodium selenite would affect the contents of those nutritional components. There were increases in the contents of proline, protein, Vc and sugar. From the results, it can be concluded that foliar application of sodium selenite clearly increased Chinese cabbage yields and improved quality.

Interestingly, we observed that exogenous selenite application showed some antioxidant properties in Chinese cabbage according to the activities of antioxidant enzymes such as POD, SOD, CAT, APX and GR. Our study coincides with the research studies of Golubkina et al. (2019) and Zimmermann and Kohrle (2002) who indicated that element Se plays an important role in immune-modulating and antioxidant properties in both mammals and plants, by defending the organism from different kinds of oxidative stress. In our study, the activities of POD, SOD, CAT, APX and GR increased due to the foliar application of sodium selenite. This result was consistent with the study of Dai et al. (2019) who also found antioxidative enzyme activities and lipid peroxidation were positive or beneficial with the application of Se.

Present study not only improved the scientific database about the effects of Se on crops, but also enhanced the application of Se in Chinese cabbage production. However, more studies are required at physiological and molecular level to reveal the mechanism about how Se affects Chinese cabbage.

\section{Conclusion}

Foliar application of sodium selenite not only significantly improved growth (Fresh weight, dry weight) by increasing the contents of photosynthetic pigments (chlorophyll $\mathrm{a}$, chlorophyll $\mathrm{b}$ and carotenoid), but also promoted Chinese cabbage nutrient quality including Vc, proline, protein, sugar and microelements such as Se, Fe and $\mathrm{Zn}$. 
Furthermore, the foliar application of Se remarkably enhanced the antioxidant system of Chinese cabbage in terms of POD, SOD, CAT, APX and GR activities. In order to reveal the mechanism about how Se affects Chinese cabbage, more studies should be conducted at physiological and molecular level

Acknowledgements. This study was supported by Shandong province key research and development project (2019GNC106121).

\section{REFERENCES}

[1] Dai, H. P., Shan, C. J., Zhao, H., Li, J. C., Jia, G. L., Jiang, H., Wu, S. Q., Wang, Q. (2015): The difference in antioxidant capacity of four alfalfa cultivars in response to $\mathrm{Zn}$. Ecotoxicology and Environmental Safety 114: 312-317.

[2] Dai, H. P., Wei, S. H., Skuza, L., Jia, G. L. (2019): Selenium spiked in soil promoted zinc accumulation of Chinese cabbage and improved its antioxidant system and lipid peroxidation. - Ecotoxicology and Environmental Safety 180: 179-184.

[3] De Medici, D., Kominkova, D., Race, M., Fabbricino, M., Souckova, L. (2019): Evaluation of the potential for caesium transfer from contaminated soil to the food chain as a consequence of uptake by edible vegetables. - Ecotoxicology and Environmental Safety 171: 558-563.

[4] Fang, Y., Wang, L., Xin, Z., Zhao, L., An, X., Hu, Q. (2008): Effect of foliar application of zinc, selenium, and iron fertilizers on nutrients concentration and yield of rice grain in China. - Journal of Agricultural and Food Chemistry 56: 2079-2084.

[5] Farooq, M., Ullah, A., Rehman, A., Nawaz, A., Nadeem, A., Wakeel, A., Nadeem, F., Siddique, K. H. M. (2018): Application of zinc improves the productivity and biofortification of fine grain aromatic rice grown in dry seeded and puddled transplanted production systems. - Field Crops Research 216: 53-62.

[6] Golubkina, N., Zamana, S., Seredin, T., Poluboyarinov, P., Sokolov, S., Baranova, H., Krivenkov, L., Pietrantonio, L., Caruso, G. (2019): Effect of Selenium Biofortification and Beneficial Microorganism Inoculation on Yield, Quality and Antioxidant Properties of Shallot Bulbs. - Plants-Basel 8(4): 102.

[7] He, L. X., Zheng, A. X., Du, B., Luo, H. W., Lu, R. H., Du, P., Chen, Y. L., Zhang, T. T., Lai, R. F., Tang, X. R. (2019): Low-concentration sodium selenite applications improve oxidation resistance of filling-stage rice. - Applied Ecology and Environmental Research 17: 989-998.

[8] Lai, R. F., Zhang, T. T., Gao, Y. H., Lu, R. H., Liu, Y. F., Zheng, A. X., Ashraf, U., Fan, P. S., Du, B., Luo, H. W., Tang, X. R. (2019): The effect of EDTA-Se with different concentrations on photosynthesis of fragrant rice (Oryza sativa L.). - Applied Ecology and Environmental Research 17: 3293-3303.

[9] Li, M., Ashraf, U., Tian, H., Mo, Z., Pan, S., Anjum, S. A., Duan, M., Tang, X. (2016): Manganese-induced regulations in growth, yield formation, quality characters, rice aroma and enzyme involved in 2-acetyl-1-pyrroline biosynthesis in fragrant rice. - Plant Physiology and Biochemistry 103: 167-175.

[10] Luo, H. W., Du, B., Zheng, A. X., Lai, R. F., You, Z. S., Wang, M., Wang, Z. M., He, L. X., Zhang, T. T., Tang, X. R. (2018): Flooding treatment restrains volunteer rice germination and seedling growth. - Applied Ecology and Environmental Research 16: 7231-7242.

[11] Ma, J. J., Ren, Y. J., Yan, L. Y. (2014): Effects of Spray Application of Lanthanum and Cerium on Yield and Quality of Chinese Cabbage (Brassica chinensis L.) Based on Different Seasons. - Biological trace element Research 160: 427-432. 


$$
-7481 \text { - }
$$

[12] Mi, B., Liu, F., Xie, L., Zhou, H., Wu, F., Dai, X. (2019): Evaluation of the uptake capacities of heavy metals in Chinese cabbage. - Ecotoxicology and Environmental Safety 171: 511-517.

[13] Rayman, M. P. (2000): The importance of selenium to human health. - Lancet (North American Edition) 356: 233-241.

[14] Su, T., Wang, W., Li, P., Zhang, B., Li, P., Xin, X., Sun, H., Yu, Y., Zhang, D., Zhao, X., Wen, C., Zhou, G., Wang, Y., Zheng, H., Yu, S., Zhang, F. (2018): A Genomic Variation Map Provides Insights into the Genetic Basis of Spring Chinese Cabbage (Brassica rapa ssp pekinensis) Selection. - Molecular Plant 11: 1360-1376.

[15] Tan, J., Zhu, W., Wang, W., Li, R., Hou, S., Wang, D., Yang, L. (2002): Selenium in soil and endemic diseases in China. - Science of the Total Environment 284: 227-235.

[16] Wu, Y. Y., Lu, X. Y., Peng, Z. K., Luo, Z. M. (2000): Effect of Se on Physiological and Biochemical Characters of Paddy Rice. - Scientia Agricultura Sinica 33(1): 100-103.

[17] Wu, Z., Xu, S., Shi, H., Zhao, P., Liu, X., Li, F., Deng, T., Du, R., Wang, X., Wang, F. (2018): Comparison of foliar silicon and selenium on cadmium absorption, compartmentation, translocation and the antioxidant system in Chinese flowering cabbage. - Ecotoxicology and Environmental Safety 166: 157-164.

[18] Xia, Y. M., Hill, K. E., Byrne, D. W., Xu, J. Y., Burk, R. F. (2005): Effectiveness of selenium supplements in a low-selenium area of China. - American Journal of Clinical Nutrition 81: 829-834.

[19] Yang, R., Liu, Y., Zhou, Z. (2017): Selenium and Selenoproteins, from Structure, Function to Food Resource and Nutrition. - Food Science and Technology Research 23: 363-373.

[20] Zhang, K., Wen, D., Cao, Z., Liang, W. (2002): Landscape ecological studies on autumnal Chinese cabbage fields in Shenyang city suburb I. Features of autumnal Chinese cabbage field patches. - Yingyong Shengtai Xuebao 13: 1615-1618.

[21] Zimmermann, M. B., Kohrle, J. (2002): The impact of iron and selenium deficiencies on iodine and thyroid metabolism: Biochemistry and relevance to public health. - Thyroid 12: 867-878. 